\title{
Re-examination of Steric Substituent Constants by Molecular Mechanics.
}

\author{
Minoru Hirota ${ }^{1,2, *}$, Kazuhisa Sakakibara ${ }^{1}$, Tomoaki Yuzuri ${ }^{1}$ and Shin-ichi Kuroda ${ }^{1}$ \\ 1 Department of Materials Science and Chemical Engineering, Faculty of Engineering, Yokohama \\ National University, Hodogaya-ku, Yokohama 240-8501, Japan \\ 2 Kanagawa High-Technology Foundation, KSP Bldg. E-201, Kawasaki 213-0012, Japan \\ Dedicated to Professor Marvin Charton \\ * Author to whom correspondence should be addressed; e-mail: mhirota@pastel.ocn.ne.jp
}

Received: 1 July 2004; in revised form: 10 October 2004 / Accepted 12 October 2004 / Published: 31 January 2005

\begin{abstract}
Statistical analysis employing the set of the steric constants $\Omega_{\mathrm{j}, \mathrm{k}}\left(R_{\mathrm{c}}\right)$ is shown to be very effective in characterizing the substituent steric effect on organic reactions. $\Omega_{\mathrm{j}, \mathrm{k}}\left(R_{\mathrm{c}}\right)$ contains several variable parameters which are useful for the diagnosis of the character of steric effect. The parameters controlling the angular, or directional, weight $(\mathrm{j}, \mathrm{k})$ and the size of reaction center atom $\mathrm{X}\left(R_{\mathrm{c}}\right)$ are varied in order to elucidate the characteristics of several steric substituent constants.
\end{abstract}

Keywords: Steric effect, Steric hindrance, Molecular mechanics, Steric substituent constant, $E_{\mathrm{s}}, v, S, \Omega_{\mathrm{s}}$

\section{Introduction}

Since the pioneering work by Taft [1], who introduced the linear free energy relationship (LFER) into the evaluation of steric substituent effects, quantitative treatment of steric substituent effects became a stimulating topic in Physical Organic Chemistry. The first steric constant $E_{\mathrm{s}}$ was defined empirically by Taft as the extension of Hammett equation [2]. By use of $E_{\mathrm{s}}$, the substituent effect on the rates can be described by the following equation: 
$\log _{10} k=\rho \sigma+\delta E_{\mathrm{s}}$

It implies that $E \mathrm{~s}$ is compatible with LFER. $E_{\mathrm{s}}$ succeeded in reproducing the steric effect of substituent very, but not perfectly, well. In this situation, a variety of steric substituent constants have been proposed by many investigators in order to better describe the steric effect. Some of them are the modifications of $E_{\mathrm{s}}$. The most important of modified $E_{\mathrm{s}}$ are $E_{\mathrm{s}}$ ' by Dubois and coworkers [3] and $E \mathrm{~s}^{\mathrm{c}}$ by Hancock and coworkers [4]. $E_{\mathrm{s}}{ }^{\prime}$ is similar to $E_{\mathrm{s}}$ but is defined on the basis of more unified reactions and over wider range of substituents. The corrected steric constant $E_{\mathrm{s}}{ }^{\mathrm{c}}$ includes an additional term in order to correct the hyperconjugation effect of $\alpha$-hydrogen atoms ( $n_{\mathrm{H}}$, number of $\alpha-\mathrm{H}$ atoms):

$$
E_{\mathrm{s}}{ }^{\mathrm{c}}=E_{\mathrm{s}}-0.306\left(3-n_{\mathrm{H}}\right)
$$

A very similar steric constant taking into consideration the hyperconjugation effect of both $\alpha-\mathrm{H}$ and $\alpha-\mathrm{C}$ was proposed by Palm and coworkers [5].

Many of other steric substituent constants were calculated by assuming appropriate models and assuming that the substituent comprises van der Waals atoms [6]. Some of them tried to correlate kinetic data with molecular geometry from molecular mechanics calculations and similar computational approach. Charton [7] proposed $v$ on the basis of the size of substituent measured by assuming that the atoms have contours of van der Waals radii $\left(R_{\mathrm{v}}\right)$. $v$ is defined as the difference between the van der Waals radii of $\mathrm{H}$ and subustituent $\mathrm{R}$. For symmetrical substituents such as $\mathrm{H}, \mathrm{Cl}$, and $\mathrm{CN}, R_{\mathrm{v}}(\max )$ were used as $R_{\mathrm{vR}}$, while $R_{\mathrm{v}}(\min )$ were employed for tetrahedral substituents such as $\mathrm{CH}_{3}$ and $\mathrm{CMe}_{3}$.

$$
v_{\mathrm{R}}=R_{\mathrm{vR}}-R_{\mathrm{vH}}=R_{\mathrm{vR}}-1.20
$$

Kier [8] defined $\Xi$ as a graph-based shape index encoding the steric effect of the substituent. $\Xi$ includes two shape indexes $\left({ }^{1} \kappa_{\alpha}\right.$ and $\left.{ }^{3} \kappa_{\alpha}\right)$ and the $\kappa_{0}$ index, and given by the following empirical equation derived on the basis of the linear correlation with $E_{\mathrm{s}}$

$$
\Xi=2{ }^{1} \kappa_{\alpha}-{ }^{3} \kappa_{\alpha}-\kappa_{0}
$$

Beckhaus [9] defined $\varphi_{\mathrm{f}}(\mathrm{R})$ as the difference of the heat of formation between the tert-butyl derivative $\mathrm{R}-\mathrm{CMe}_{3}$ and those of the methyl derivative $\mathrm{R}-\mathrm{CH}_{3}$. As $\mathrm{R}-\mathrm{CMe}_{3}$ has a bulky tert-butyl group at the front side of $\mathrm{R}, \varphi_{\mathrm{f}}$ is expected to measure the enthalpy increase by the F-strain.

$$
\varphi_{\mathrm{f}}(\mathrm{R})=\Delta H_{\mathrm{f}}^{0}\left(\mathrm{R}-\mathrm{CMe}_{3}\right)-\Delta H_{\mathrm{f}}^{0}\left(\mathrm{R}-\mathrm{CH}_{3}\right)
$$

Meyer [10] assumed that the steric effect can be evaluated by the combination of shape descriptor $G$ and bulkiness descriptor $V^{\mathrm{a}}$ both estimated on the basis of van der Waals molecules. $G$ is calculated as the ratio of surface area of substituent to its volume, and $V^{\mathrm{a}}$ is the volume of substituent within $0.3 \mathrm{~nm}$ from the reaction center. Chauvin and Kagan [11] defined $S$ and $S$ ' as a nonempirical steric index based on relatively simple calculations. Both $S$ and $S^{\prime}$ are essentially the solid angle of the space behind the substituent. They reported a quasi-linear relation between $S$ and $E_{\mathrm{s}}$ '; however correlation is not very good. In contrast, $1 / S^{\prime}$ could be linearly correlated excellently with $E_{\mathrm{s}}$ '. 
The present authors proposed $\Omega_{\mathrm{s}}$ as an accurate steric substituent constant based on molecular mechanics calculations on the model $\mathrm{R}-\mathrm{X}$ molecule [12]. If we assume that a light source is placed at the reaction-center-atom $(\mathrm{X})$ and if the shadow of the substituent group $(\mathrm{R})$ is projected on the surrounding sphere (its radius $R_{\mathrm{S}}=0.4 \mathrm{~nm}$ ), the proportion of the shadow area can be a measure of the steric hindrance effect caused by R. As easily understood from the definition of $\Omega_{\mathrm{s}},\left(1-\Omega_{\mathrm{s}}\right)$ should be proportional to the frequency factor of the Arrhenius equation and, hence, $\log _{10}\left(1-\Omega_{\mathrm{s}}\right)$ should be used in the LFER equations. As a complex substituent group has more than one unequal conformer, $\Omega_{\mathrm{s}}$ was defined as the population-weighted sum of normalized solid angles of shadow area over these conformers. In earlier stage of our $\Omega_{\mathrm{s}}$, the radii of both reaction-center-atom $\mathrm{X}\left(R_{\mathrm{c}}\right)$ and reagent $\mathrm{Y}\left(R_{\mathrm{r}}\right)$ are assumed to be null in order to simplify the model and to curtail the time required for the calculation [12a,b]. However, we were aware that this approximation can cause a serious defect, since the performance of $\Omega_{\mathrm{s}}$ was shown to be considerably dependent on the radius of $\mathrm{X}\left(R_{\mathrm{c}}\right)$ [13]. $\Omega_{\mathrm{S}}$ calculated by assuming larger $R_{\mathrm{c}}$ is shown to reproduce the steric effect on the reactions including the unshared electron pair of the attached atom X. In contrast, $\Omega_{\mathrm{s}}$ based on smaller $R_{\mathrm{c}}$ is good for the substituent effect on cationic reaction center.

Another modification of $\Omega_{\mathrm{s}}$ stands on the calculation using angular weight-functions $f_{\mathrm{jk}}(\theta, \phi)[13 \mathrm{~b}]$. Thus:

$$
\begin{gathered}
\Omega_{\mathrm{j}, \mathrm{k}}\left(r_{\mathrm{c}}\right)=(1 / 4 \pi) \Sigma \sigma\left(r_{\mathrm{c}}\right) f_{\mathrm{jk}}(\theta, \phi) \Delta \omega \quad \sigma=1 \text { for the shadow area. } \\
\sigma=0 \text { for the open area. }
\end{gathered}
$$

In this equation the summation was carried out over every small increment $(\Delta \omega)$ of the whole solid angle. The calculated $\Omega_{\mathrm{j}, \mathrm{k}}\left(R_{\mathrm{c}}\right)$ exaggerates steric hindrance to the access of the reagent $\mathrm{Y}$ from the direction $(\theta, \phi)$ corresponding to large $f_{\mathrm{jk}}(\theta, \phi)$ values; thus it is expected to be useful in estimating the preferable direction to the attack of the reagent. An important point to be remarked is the fact that all weight-functions $f_{\mathrm{jk}}(\theta, \phi)$ are not orthogonal but rather closely correlated to each other. Weight-functions are designed so as to reproduce the directional preference of the access of Y. For example, the attack of $\mathrm{Y}$ to substituted carbonyl compounds $\mathrm{R}-\mathrm{C}=\mathrm{O}$ is expected to take place preferably when $\mathrm{Y}$ comes from the direction of the axis of p-orbital of $\mathrm{X}$ (carbonyl $\mathrm{C}$, in this case). In other words, $\Omega_{7,6}$ calculated on the basis of p-shaped weight-function $f_{7,6}(\theta, \phi)\left[=\left(f_{7,6} \times f_{7,6}\right)^{-1 / 2}\right]$ is expected to reproduce the substituent effect best.

\section{Methods}

In the present work, we wish to show how the performance of the steric parameters are perturbed by the variations of $R_{\mathrm{c}}$ and $f_{\mathrm{jk}}(\theta, \phi)$ and to discuss the results from the mechanism of the reactions involved. The weight-functions employed in this investigation are given together with short explanations in Table 1. All $\Omega_{\mathrm{s}}$ calculations were carried out by MM3 [16] using the following parameters: $\mathrm{X}=\mathrm{COOH}(\mathrm{X}=$ $\mathrm{CH}_{3}$ in the cases of $\Omega_{1,12}$ and $\left.\Omega_{1,13}\right), T=313.15 \mathrm{~K}$ (in the calculation of conformer distribution of R), $R_{\mathrm{s}}($ radius of screen sphere $)=0.4 \mathrm{~nm}, R_{\mathrm{r}}($ radius of reagent $\mathrm{Y})=0.05 \mathrm{~nm}$. 
Table 1. Weight-functions $f_{\mathrm{jk}}(\theta, \phi)[15]$

\begin{tabular}{|c|c|l|l|}
\hline $\mathbf{j}$ & $\mathbf{k}$ & \multicolumn{1}{|c|}{$\boldsymbol{f}_{\mathbf{j k}}(\theta, \phi)}$. & \multicolumn{1}{c|}{ Weighted direction } \\
\hline 1 & 1 & $=1$ & Isotropic \\
2 & 1 & $=(1+\cos \theta) / 2$ & Backward (R side) \\
3 & 1 & $=(1-\cos \theta) / 2$ & Front side (X side) \\
6 & 1 & $=(1+\cos 2 \theta) / 2$ & Direction of R-X bond axis \\
7 & 1 & $=(1-\cos 2 \theta) / 2$ & Vertical to R-X bond axis \\
10 & 1 & $=(1+\cos 3 \theta) / 2$ & Front-side cone(apical angle $\left.120^{\circ}\right)$ \\
11 & 1 & $=(1-\cos 3 \theta) / 2$ & Backward cone(apical angle $\left.120^{\circ}\right)$ \\
1 & 6 & $=(1+\cos 2 \phi) / 2$ & Perpendicular to molecular plane (in R-COOH model) $)$ \\
1 & 7 & $=(1-\cos 2 \phi) / 2$ & In-plane direction (in R-COOH model) \\
1 & 12 & $=(1+\sin 3 \phi) / 2$ & Eclipsed to three $\mathrm{C}_{\alpha}$ substituent \\
1 & 13 & $=(1-\sin 3 \phi) / 2$ & In between (staggered) to three $\mathrm{C}_{\alpha}$ substituent \\
7 & 6 & $=\left[f_{7,1} \times f_{1,6}\right]^{1 / 2}$. & $\pi$-Orbital-like direction at X \\
\hline
\end{tabular}

$\Omega_{\mathrm{j}, \mathrm{k}}$ was calculated over a range of the size of X (from $R_{\mathrm{c}}=0.03$ to $0.12 \mathrm{~nm}$, usually). Then, $\Omega_{\mathrm{j}, \mathrm{k}}$ constants for various $\mathrm{j}$ and $\mathrm{k}$ were applied to the linear regression analysis of steric substituent effect of some typical reactions in order to examine its effectiveness. For this purpose, the $\log _{10} k$ were plotted against $\log _{10}\left[1-\Omega_{\mathrm{j}, \mathrm{k}}\left(R_{\mathrm{c}}\right)\right]$ assuming the linear regression (eq. 7):

$$
\log _{10} k=a \log _{10}\left[1-\Omega_{\mathrm{j}, \mathrm{k}}\left(R_{\mathrm{c}}\right)\right]+b
$$

The correlation coefficient $r$ was calculated in order to monitor their reliability and to search out the best-fit $\Omega_{\mathrm{j}, \mathrm{k}}\left(R_{\mathrm{c}}\right)$. By comparing the $r$ values within a set of correlations using the similar weight-functions (both $\mathrm{j}$ and $\mathrm{k}$ are the same with each other) at various $R_{\mathrm{c}}$ by drawing the $r \mathrm{vs} . R_{\mathrm{c}}$ plot, the most probable $R_{\mathrm{c}}$ was picked up. Here the peak of the $r$ vs. $R_{\mathrm{c}}$ plot corresponds to the most probable $R_{\mathrm{c}}$.

\section{Results and Discussion}

Analysis of Steric Effects on Some Important Reactions

A few typical results are illustrated as the $r$ vs. $R_{\mathrm{c}}$ plots in Figure 1 . Similar $r$ vs. $R_{\mathrm{c}}$ plots gave the most probable $R_{\mathrm{c}}$ for the other reactions. The best correlation coefficient $(r)$ and the radius of X $\left(R_{\mathrm{c}}\right)$ at which the best performance of $\Omega_{\mathrm{j}, \mathrm{k}}\left(R_{\mathrm{c}}\right)$ is achieved are collected in Table 2. We could deduce several important features which justify the application of this sort of analysis to the stereochemical problems of organic reactions.

In the same row of Table 2, the same data of reaction rates are treated using different $\Omega_{\mathrm{j}, \mathrm{k}}\left(R_{\mathrm{c}}\right)$; therefore the most probable $R_{\mathrm{c}}$ should be the same within a row. Some of $\Omega_{\mathrm{j}, \mathrm{k}}\left(R_{\mathrm{c}}\right)$ 's are not very sensitive to the change in $R_{\mathrm{c}}$, resulting a broad peak in the $r$ vs. $R_{\mathrm{c}}$ plots in Figure 1 . The $r$ vs. $R_{\mathrm{c}}$ curves for the same reaction but for different set of $\mathrm{j}, \mathrm{k}$ are similar to each other except for a relatively few anomalous ones. 
Figure 1. The $R$ vs. $r_{\mathrm{c}}$ Plots for Some Reactions in Table 2.

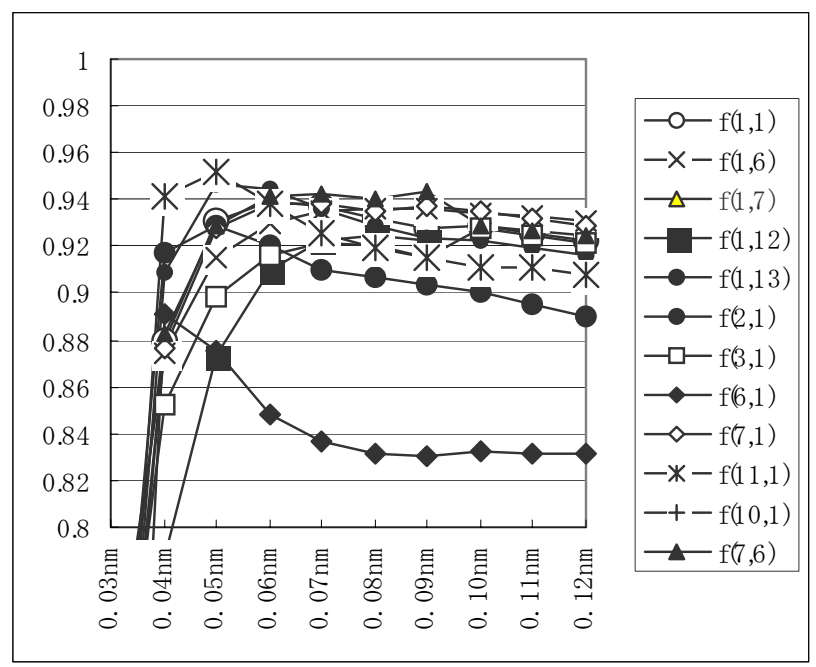

(A) Reaction 1.

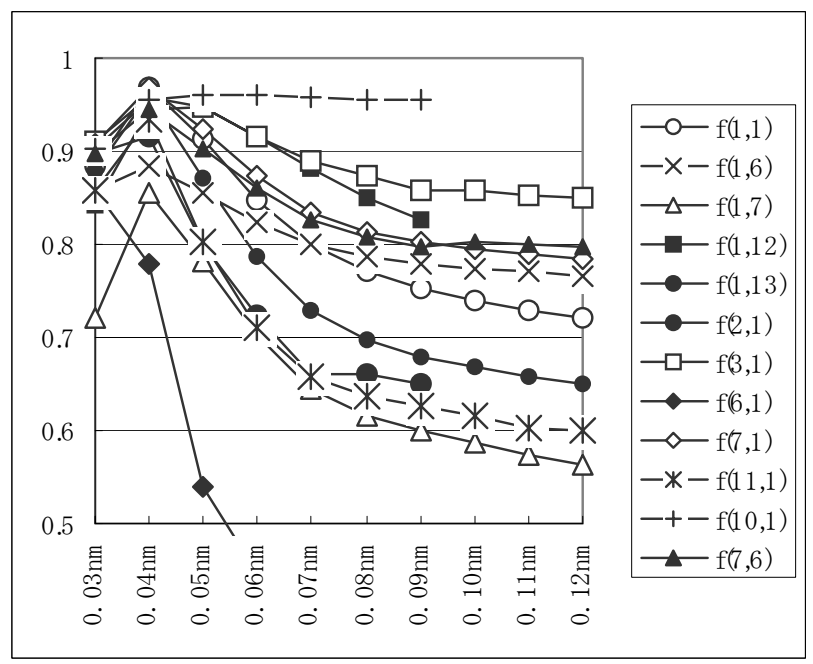

(B) Reaction 5.

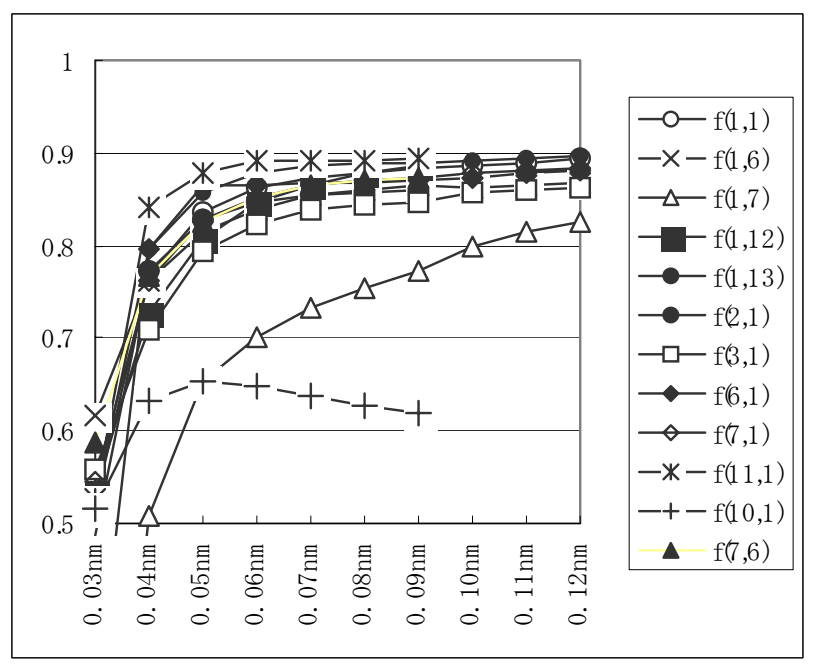

(C) Reaction 9. 
Figure 1. Cont.

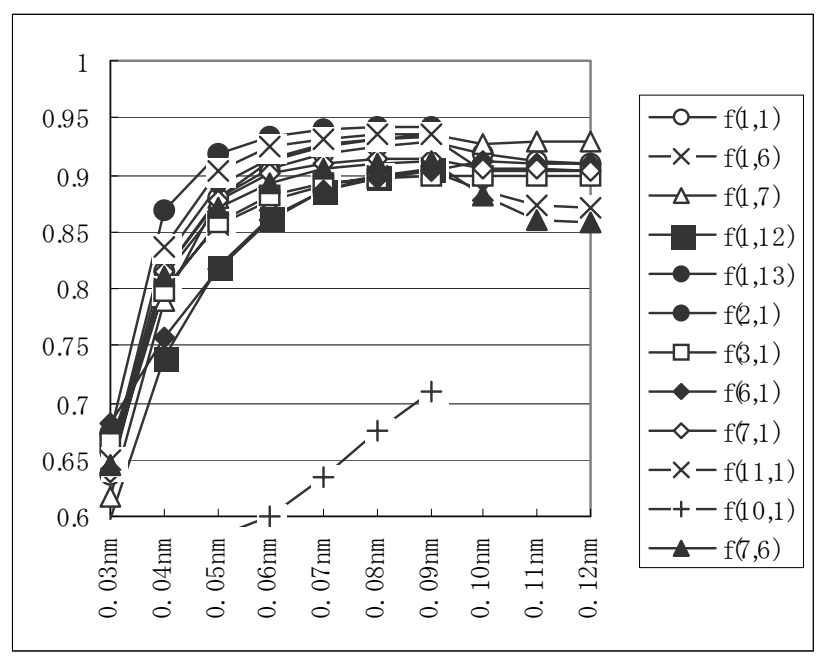

(D) Biological Activity 13 .

As can be certified by the data in Table 2 and also supported by the similarity of the curves in Figure 1 , the best fit $R_{\mathrm{c}}$ value in the same row is on the whole constant irrespective of the variation in angular parameters ( $\mathrm{j}$ and $\mathrm{k}$ ) of $\Omega_{\mathrm{j}, \mathrm{k}}\left(R_{\mathrm{c}}\right)$. This can be a favorable evidence for the reliability of $R_{\mathrm{c}}$.

However, the results from this sort of computational study should be critically examined from the viewpoint of theoretical consistency. As pointed out by a preliminary work $[12,13]$ and shown more thoroughly in the present work, the $R_{\mathrm{c}}$ values from our calculations are in agreement with the general trend that the cationic center is smaller than the anionic center. After all, the reliability of $R_{\mathrm{c}}$ as a measure of the size of the reaction-center-atom is guaranteed. This enables us to estimate the relative size of the reaction center atom $(\mathrm{X})$ by this method.

Table 2. The best $r$ (upper) and the corresponding $R_{\mathrm{c}} / \mathrm{nm}$ (lower) obtained from the $r \mathrm{vs} . R_{\mathrm{c}}$ curves based on $\log _{10} k=a \log _{10}\left[1-\Omega_{\mathrm{j}, \mathrm{k}}\left(r_{\mathrm{c}}\right)\right]+b$ correlations of various reactions.*)

\begin{tabular}{|c|c|c|c|c|c|c|c|c|c|c|c|c|}
\hline $\begin{array}{l}\text { No. } \\
\text { React } \\
\end{array}$ & $\begin{array}{l}j=1 \\
k=1\end{array}$ & $\begin{array}{l}j=2 \\
k=1\end{array}$ & $\begin{array}{l}j=3 \\
k=1\end{array}$ & $\begin{array}{l}j=6 \\
k=1\end{array}$ & $\begin{array}{l}j=7 \\
k=1\end{array}$ & $\begin{array}{l}j=10 \\
k=1\end{array}$ & $\begin{array}{l}j=11 \\
k=1\end{array}$ & $\begin{array}{l}j=1 \\
k=6\end{array}$ & $\begin{array}{l}j=1 \\
k=7\end{array}$ & $\begin{array}{l}j=1 \\
k=12\end{array}$ & $\begin{array}{l}j=1 \\
k=13\end{array}$ & $\begin{array}{l}j=7 \\
k=6\end{array}$ \\
\hline \multirow[t]{2}{*}{1} & & 947 & & 0801 & & 0.769 & & 0.936 & 0.758 & & 0.929 & 0.943 \\
\hline & & 0.05 & 0.07 & 0.04 & 0.05 & 0.05 & 0.04 & 0.08 & 0.06 & 0.08 & 0.05 & 0.09 \\
\hline \multirow[t]{2}{*}{2} & & 0.973 & $00=0$ & 0.854 & 0.966 & 0.851 & $\underline{0.977}$ & & 0.748 & 0.968 & 0.914 & 0.976 \\
\hline & & 0.05 & 0.07 & 0.05 & 0.06 & 0.05 & 0.04 & 0.05 & 0.09 & 0.06 & 0.05 & 0.06 \\
\hline \multirow[t]{2}{*}{3} & & & & & & 0.766 & & & & & & 0.979 \\
\hline & & 0.06 & 0.06 & 0.04 & 0.07 & 0.05 & 0.05 & 0.09 & 0.06 & 0.09 & 0.05 & 0.09 \\
\hline \multirow[t]{2}{*}{4} & $\underline{0.966}$ & 0.959 & & 0.790 & $\underline{0.966}$ & 0.956 & 0.935 & 0.891 & 0.843 & 0.949 & 0.912 & 0.944 \\
\hline & 0.04 & 0.04 & 0.04 & 0.04 & 0.04 & 0.05 & 0.04 & 0.04 & 0.04 & 0.05 & 0.04 & 0.04 \\
\hline \multirow[t]{2}{*}{5} & & 0.961 & 0.957 & 0.780 & $\underline{0.969}$ & 0.961 & 0.935 & 0.885 & 0.856 & 0.947 & 0.915 & 0.944 \\
\hline & 0.04 & 0.04 & 0.04 & 0.04 & 0.04 & 0.05 & 0.04 & 0.04 & 0.04 & 0.05 & 0.04 & 0.04 \\
\hline
\end{tabular}


Table 2. Cont.

\begin{tabular}{|c|c|c|c|c|c|c|c|c|c|c|c|c|}
\hline $\begin{array}{l}\text { No. } \\
\text { React }\end{array}$ & $\begin{array}{l}j=1 \\
k=1\end{array}$ & $\begin{array}{l}j=2 \\
k=1\end{array}$ & $\begin{array}{l}j=3 \\
k=1\end{array}$ & $\begin{array}{l}j=6 \\
k=1\end{array}$ & $\begin{array}{l}j=7 \\
k=1\end{array}$ & $\begin{array}{l}\mathbf{j}=\mathbf{1 0} \\
\mathrm{k}=\mathbf{1}\end{array}$ & $\begin{array}{l}j=11 \\
k=1\end{array}$ & $\begin{array}{l}j=1 \\
k=6\end{array}$ & $\begin{array}{l}j=1 \\
k=7\end{array}$ & $\begin{array}{l}j=1 \\
k=12\end{array}$ & $\begin{array}{l}j=1 \\
k=13\end{array}$ & $\begin{array}{l}j=7 \\
k=6\end{array}$ \\
\hline \multirow[t]{2}{*}{6} & & 850 & 0.834 & 0.676 & 0.849 & 0.774 & 0.830 & & 0.715 & .883 & 0.684 & 0.862 \\
\hline & & 0.04 & 0.04 & & & & 0.04 & 0.04 & 0.04 & 0.05 & 0.06 & 0.04 \\
\hline \multirow[t]{2}{*}{7} & & 0700 & 0005 & 0.736 & 0015 & 0.565 & 0.822 & 0.771 & $\underline{0.848}$ & 0.817 & 0.784 & 0.831 \\
\hline & & 0.04 & 0.05 & 0.04 & 0.05 & 0.05 & 0.04 & 0.05 & 0.05 & 0.06 & 0.04 & 0.04 \\
\hline \multirow[t]{2}{*}{8} & & & & & & 0.330 & & & & & $\underline{0.938}$ & 0.900 \\
\hline & & 0.12 & & & & & & & & & & 0.09 \\
\hline \multirow[t]{2}{*}{9} & & & & & & & & & & & & 0.873 \\
\hline & & & & $>0.12$ & & 0.05 & 0.09 & 0.09 & 0.08 & $>0.09$ & 0.09 & 0.09 \\
\hline \multirow[t]{2}{*}{10} & & & & & & & & & & & $\underline{0.941}$ & 0.820 \\
\hline & & & & & & & & & 0.09 & 0.09 & 0.06 & 0.08 \\
\hline \multirow[t]{2}{*}{11} & & & & & & & & & & & & 0.884 \\
\hline & & 0.07 & 0.08 & & & 0.06 & & & 0.09 & 0.09 & 0.06 & 0.08 \\
\hline \multirow[t]{2}{*}{12} & 0.803 & 0.845 & 0.700 & $\underline{0.902}$ & 0.764 & 0.321 & 0.866 & 0.710 & 0.841 & 0.711 & 0.854 & 0.737 \\
\hline & 0.12 & 0.12 & 0.11 & 0.06 & 0.11 & 0.05 & 0.09 & 0.12 & 0.12 & $>0.09$ & $>0.09$ & 0.09 \\
\hline \multirow[t]{2}{*}{13} & 0.928 & 0.933 & 0.900 & 0.911 & 0.915 & 0.935 & 0.710 & 0.903 & 0.935 & 0.906 & 0.942 & 0.913 \\
\hline & مQ & 0.09 & 0.10 & 0.10 & 0.09 & 0.09 & $>0.09$ & 0.09 & 0.09 & $>0.09$ & 0.09 & 0.09 \\
\hline
\end{tabular}

*) The best $r$-value through the same row is given by underlined bold figures; other better $r$-values are marked by bold figures.

[Reactions]

1) $\mathrm{RCOOH}+\mathrm{MeOH}$ in $\mathrm{MeOH} / \mathrm{HCl}$ at $50^{\circ} \mathrm{C}[17 \mathrm{a}]$.

2) $\mathrm{RCOOEt}+\mathrm{H}_{2} \mathrm{O}$ in $\mathrm{AcMe}$ at $20^{\circ} \mathrm{C}[17 \mathrm{~b}]$.

3) $\mathrm{RCOOEt}+\mathrm{OH}-$ in $85 \% \mathrm{EtOH}$ at $50^{\circ} \mathrm{C}[17 \mathrm{c}]$.

4) $\mathrm{RCONH}_{2}+\mathrm{H}_{3} \mathrm{O}^{+}$in $\mathrm{H}_{2} \mathrm{O}$ at $75^{\circ} \mathrm{C}[17 \mathrm{~d}]$.

5) $\mathrm{RCONH}_{2}+\mathrm{H}_{3} \mathrm{O}^{+}$in $\mathrm{H}_{2} \mathrm{O}$ at $85^{\circ} \mathrm{C}[17 \mathrm{~d}]$.

6) $\mathrm{RCONH}_{2}+\mathrm{OH}^{-}$in $\mathrm{H}_{2} \mathrm{O}$ at $75^{\circ} \mathrm{C}[17 \mathrm{e}]$.

7) $\mathrm{RCONH}_{2}+\mathrm{OH}^{-}$in $\mathrm{H}_{2} \mathrm{O}$ at $95^{\circ} \mathrm{C}[17 \mathrm{e}]$.

8) $\mathrm{PhCH}_{2} \mathrm{COOR}+\mathrm{OH}^{-}$in $\mathrm{H}_{2} \mathrm{O} / \mathrm{AcMe}$ at $25^{\circ} \mathrm{C}[17 \mathrm{f}]$.

9) $\mathrm{ROH}+p-\mathrm{NO}_{2} \mathrm{C}_{6} \mathrm{H}_{4} \mathrm{COCl}$ in $\mathrm{Et}_{2} \mathrm{O}$ at $25^{\circ} \mathrm{C}[17 \mathrm{~g}, \mathrm{~h}]$.

10) $\mathrm{CH}_{3} \mathrm{CONHR}+\mathrm{H}_{3} \mathrm{O}^{+}$in $\mathrm{H}_{2} \mathrm{O}$ at $75^{\circ} \mathrm{C}$ [17i].

11) $\mathrm{RPO}(\mathrm{OEt})\left(\mathrm{O} \mathrm{C}_{6} \mathrm{H}_{4} \mathrm{NO}_{2}-p\right)+\mathrm{OH}^{-}$in $\mathrm{H}_{2} \mathrm{O}$ at $37.5^{\circ} \mathrm{C}[17 \mathrm{j}]$.

12) $\mathrm{RMe}_{2} \mathrm{SiCl}+\mathrm{H}_{2} \mathrm{O}$ in $\mathrm{H}_{2} \mathrm{O} /$ dioxane at $25^{\circ} \mathrm{C}[17 \mathrm{k}, 1]$.

13) Toxicity $\left(\mathrm{LC}_{95}\right)$ of 5-phenoxy-2-pyridiyl 3-(4-substituted(R) phenyl)2,2-dimethylcyclopropane- carboxylates towards a Japanese acarus (Namihadani) [17m].

Next we wish to make a brief remark on the difference in the performance of variously weighted $\Omega_{\mathrm{j}, \mathrm{k}}\left(R_{\mathrm{c}}\right)$. In principle, $\Omega_{\mathrm{j}, \mathrm{k}}\left(R_{\mathrm{c}}\right)$ has been designed so as to exaggerate the direction of the access of $\mathrm{Y}$ by use of the weight-function $f_{\mathrm{jk}}(\theta, \phi)$. Thus, the best-fit $\Omega_{\mathrm{j}, \mathrm{k}}\left(R_{\mathrm{c}}\right)$ should reproduce the direction of access of 
Y most faithfully. However, the situation is a little more complicated because the space weighted by $f_{\mathrm{jk}}(\theta, \phi)$ can overlap the directions of geometrical arrangement of atoms consisting of $\mathrm{R}$. As a result, the effective range $\left(D=\left|\left[\Omega_{\mathrm{j}, \mathrm{k}}\right]_{\max }-\left[\Omega_{\mathrm{j}, \mathrm{k}}\right]_{\min }\right|\right)$ of $\Omega_{\mathrm{j}, \mathrm{k}}$ becomes large when the weighted space overlaps the direction of substituent groups. Most of the constituent atoms of $\mathrm{R}$ occupy the space exaggerated by $f_{2,1}(\theta, \phi)$, and three substituent groups $\left(\mathrm{R}^{1}, \mathrm{R}^{2}\right.$ and $\left.\mathrm{R}^{3}\right)$ on $\mathrm{C}_{\alpha}$ of $\mathrm{R}$ stretch towards the most weighted space of $f_{11,1}(\theta, \phi)$. This implies that the steric hindrance effect should be predominantly governed and, thus best described, by $\Omega_{2,1}\left(R_{\mathrm{c}}\right)$ and $\Omega_{11,1}\left(R_{\mathrm{c}}\right)$. The effective ranges of these $\Omega_{\mathrm{j}, \mathrm{k}}\left(\right.$ at $\left.R_{\mathrm{c}}=0.09 \mathrm{~nm}\right)$ are 0.388 and 0.479 , respectively, and the largest two among all $\Omega_{\mathrm{j}, \mathrm{k}}$ in Table 1 . In contrast, the $\Omega_{6,1}$ exaggerating the direction of $\mathrm{C}_{\alpha}-\mathrm{X}$ axis has the smallest $D(=0.102)$.

Hydrolysis of amides(Reactions $4-7$ in Table 2) showed a very characteristic common feature taking relatively short optimal $R_{\mathrm{c}}$-values at about $0.05 \mathrm{mn}$; their $r$ 's of the $\log k \mathrm{vs}$. $\Omega_{\mathrm{j}, \mathrm{k}}\left(R_{\mathrm{c}}\right)$ correlation abruptly lowering as the increase in $R_{\mathrm{c}}$ (Reaction 5 in Fig. 1 ). The optimal $R_{\mathrm{c}}$-values for hydrolyses of carboxylate esters and esterification of carboxylic acids become a little larger and within the range from 0.04 to $0.07 \mathrm{~nm}$ in most cases. The version of $\Omega_{2,1}$ with $R_{\mathrm{c}}=0.05 \mathrm{~nm}$ showed excellent performance throughout the esterification/hydrolysis reactions of carboxylic derivatives ( $\mathrm{R}-\mathrm{CO}-\mathrm{Z})$. The $\mathrm{R}-\mathrm{N}$ and $\mathrm{R}-\mathrm{O}$ containing molecules which reacts on their unshared electrons pair have longer optimal $R_{\mathrm{c}}$ (from 0.08 to $0.12 \mathrm{~nm}$ ), reflecting the character of rather loosely bound electrons in participation of the reaction. The $R_{\mathrm{c}}$ values from the hydrolysis of $p$-nitrophenyl esters of phosphonic acids are considerably scattered in the range from 0.06 to $0.11 \mathrm{~nm}$. In this case, $\mathrm{P}-\mathrm{C}_{\alpha}$ bond is considerably longer than the model $\mathrm{R}-\mathrm{COOH}$ molecule; which might cause some inconsistency among the data.

\section{Evaluation of Typical Steric Substituent Constants by Using $\Omega_{\mathrm{j}, \mathrm{k}}\left(R_{\mathrm{c}}\right)$}

Molecular mechanics is known as a facile and effective means to precisely evaluate the steric effect. Thus, we next applied the analysis using $\Omega_{\mathrm{j}, \mathrm{k}}\left(R_{\mathrm{c}}\right)$ to the critical re-examination of conventionally used steric constants.. Results of the analysis on $E_{\mathrm{s}}$ are illustrated in Figure 2, and the summary of the best $r$ and the corresponding best-fit $R_{\mathrm{c}}$ for several steric substituent constants are given in Table 3 .

The most frequently used steric constants $E_{\mathrm{s}}[1]$ and $E_{\mathrm{s}}$ [3] are both defined on the basis of the rates of ester-hydrolysis/carboxylic-acid-esterification reactions. These two steric constants are expected to accurately reproduce the steric hindrance judging from higher $r$ for the $C$ vs. $\Omega_{\mathrm{j}, \mathrm{k}}\left(R_{\mathrm{c}}\right)$ correlations than those for the individual reactions in Table 2. The pattern of variation of best-fit $r$ among the set of weighted $\Omega_{\mathrm{j}, \mathrm{k}}\left(R_{\mathrm{c}}\right)$ correlations is very similar to the carboxylate hydrolysis and esterification reactions (Reactions $1-3$ ). The similarity must come from their original definition; i. e., the most characteristic feature common with the hydrolysis/esterification is the relatively short optimal $R_{\mathrm{c}}$ value. The analysis showed that these steric constants $\left(E_{\mathrm{s}}\right.$ and $E_{\mathrm{s}}$ ') fit most accurately to the reactions of hydrolysis/esterification of carboxylic acid derivatives and related compounds.

Hancock's $E_{\mathrm{s}}{ }^{\mathrm{c}}$ [4] was proposed as a modification of $E_{\mathrm{s}}$ in which the contribution by the hyperconjugation of $\alpha-\mathrm{H}$ of substituent $\mathrm{R}$ is taken into account. Hancock's modification on $E_{\mathrm{s}}$ is equivalent to the reduction of steric hindrance contribution by $\alpha-\mathrm{H}$ atom(s) relative to other atoms located farther from X. From purely geometrical aspect, the relative contribution of $\alpha$-atoms to the total steric hindrance effect should decrease as the size of reaction-center-atom $\mathrm{X}$ becomes large. This is 
reflected to the larger optimal $R_{\mathrm{c}}$ values for $E_{\mathrm{s}}{ }^{\mathrm{c}}$. This modification should favor for the general use of $E_{\mathrm{s}}{ }^{\mathrm{c}}$ in a wide range of reactions including the evaluation of biological activity (for example, run 13 of Table 2 ), since larger $R_{\mathrm{c}}$ is expected in nucleophilic reactions on $\mathrm{X}$ and in biological phenomena involving the unshared electrons on $\mathrm{X}$ atoms.

Figure 2. The $R$ vs. $r_{\mathrm{c}}$ plots for $E_{\mathrm{s}}($ Taft $)$.

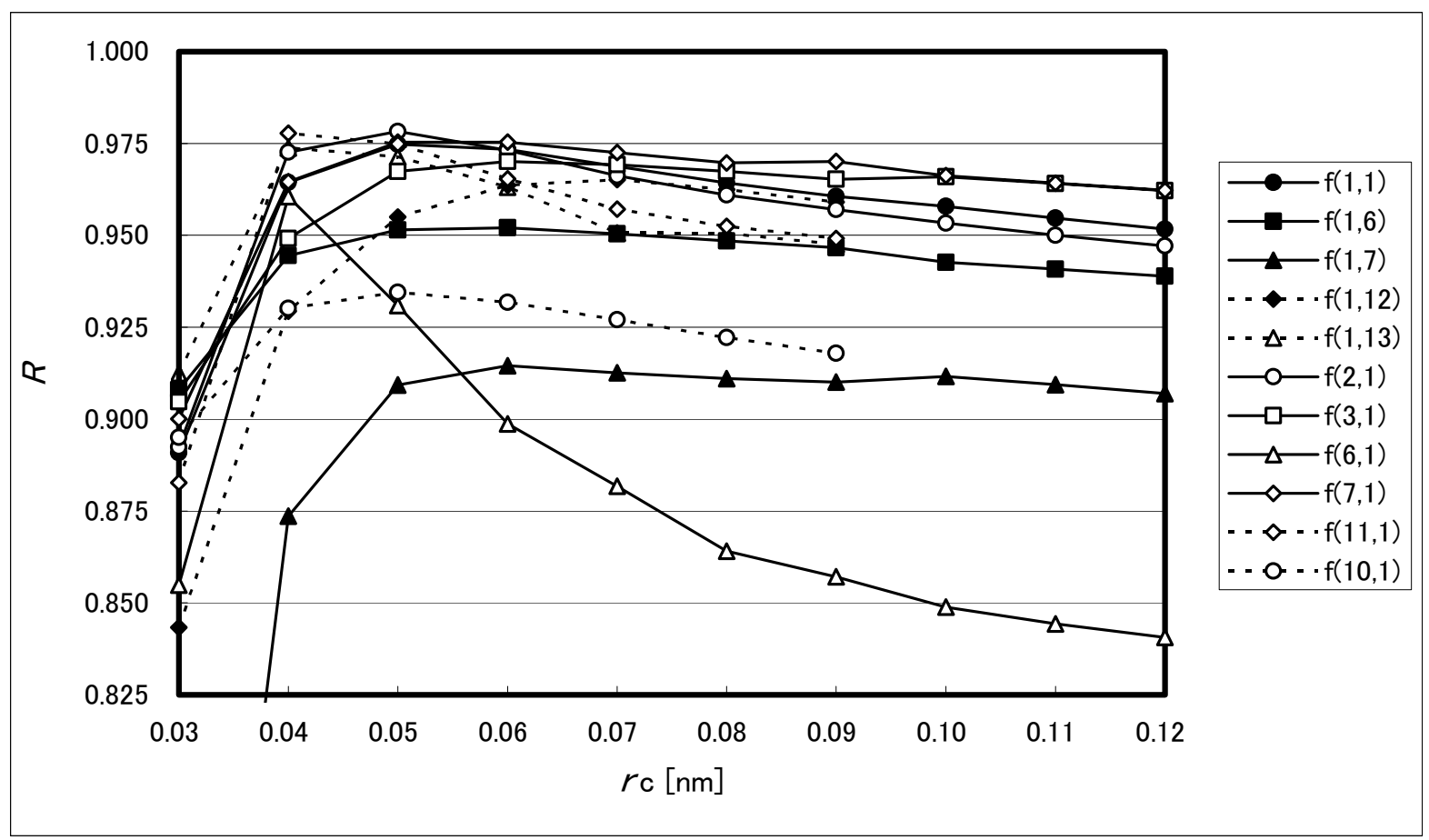

Table 3. The best correlation coefficient $r$ (upper) and $R_{\mathrm{c}} / \mathrm{nm}$ (lower) obtained on the basis of $C=a \log _{10}\left[1-\Omega_{\mathrm{j}, \mathrm{k}}\left(r_{\mathrm{c}}\right)\right]+b$ correlations for various steric substituent constants $(C)[1-11]$.

\begin{tabular}{|c|c|c|c|c|c|c|c|c|c|c|c|c|}
\hline $\begin{array}{c}\text { Steric } \\
\text { Const } \\
\quad C \\
\end{array}$ & $\begin{array}{l}j=1 \\
k=1\end{array}$ & $\begin{array}{l}j=2 \\
k=1\end{array}$ & $\begin{array}{l}j=3 \\
k=1\end{array}$ & $\begin{array}{l}j=6 \\
k=1\end{array}$ & $\begin{array}{l}\mathbf{j}=7 \\
k=1\end{array}$ & $\begin{array}{c}\mathbf{j}=10 \\
\mathrm{k}=1\end{array}$ & $\begin{array}{l}j=11 \\
k=1\end{array}$ & $\begin{array}{l}j=1 \\
k=6\end{array}$ & $\begin{array}{l}j=1 \\
k=7\end{array}$ & $\begin{array}{c}j=1 \\
k=12\end{array}$ & $\begin{array}{c}\mathbf{j}=1 \\
k=13\end{array}$ & $\begin{array}{l}j=7 \\
k=6\end{array}$ \\
\hline \multirow[t]{2}{*}{$E_{\mathrm{s}}$} & 975 & 978 & 970 & 0.961 & 975 & 0.934 & $\underline{0.978}$ & 0.952 & 0.915 & 0.965 & 0.973 & 0.968 \\
\hline & 0.05 & 0.05 & 0.06 & 0.04 & 0.05 & 0.05 & 0.04 & 0.06 & 0.06 & 0.07 & 0.04 & 0.06 \\
\hline \multirow[t]{2}{*}{$E_{\mathrm{s}}}$, & 0.980 & $\underline{0.984}$ & 0.977 & 0.982 & 0.982 & 0.962 & $\underline{0.984}$ & 0.966 & 0.959 & 0.971 & 0.983 & 0.968 \\
\hline & 0.05 & 0.05 & 0.05 & 0.04 & 0.05 & 0.05 & 0.04 & 0.05 & 0.05 & 0.06 & 0.04 & 0.06 \\
\hline \multirow[t]{2}{*}{$E_{\mathrm{s}}^{\mathrm{c}}$} & 0.975 & 0.977 & 0.966 & 0.968 & 0.973 & 0.903 & 0.974 & 0.951 & 0.925 & 0.958 & $\underline{0.979}$ & 0.973 \\
\hline & 0.06 & 0.06 & 0.07 & 0.04 & 0.07 & 0.05 & 0.06 & 0.07 & 0.08 & 0.08 & 0.05 & 0.07 \\
\hline \multirow[t]{2}{*}{$v$} & 0.957 & 0.955 & 0.968 & 0.844 & $\underline{0.970}$ & 0.931 & 0.950 & 0.952 & 0.907 & 0.951 & 0.955 & 0.965 \\
\hline & 0.06 & 0.06 & 0.07 & 0.10 & 0.06 & 0.07 & 0.07 & 0.06 & 0.10 & 0.07 & 0.05 & 0.06 \\
\hline \multirow[t]{2}{*}{$\varphi_{\mathrm{f}}$} & 0.969 & 0.971 & 0.964 & $\underline{0.983}$ & 0.970 & 0.917 & 0.966 & 0.955 & 0.954 & 0.956 & 0.974 & 0.973 \\
\hline & 0.07 & 0.06 & 0.08 & 0.04 & 0.08 & 0.05 & 0.05 & 0.09 & 0.06 & 0.08 & 0.06 & 0.08 \\
\hline \multirow[t]{2}{*}{$\Xi$} & 0.977 & 0.982 & 0.964 & 0.979 & 0.975 & 0.862 & 0.982 & 0.970 & 0.902 & 0.958 & $\underline{0.986}$ & 0.973 \\
\hline & 0.11 & 0.08 & 0.12 & 0.04 & $>0.12$ & 0.05 & 0.07 & $>0.12$ & 0.11 & $>0.09$ & 0.07 & 0.09 \\
\hline
\end{tabular}


Table 3. Cont.

\begin{tabular}{|c|c|c|c|c|c|c|c|c|c|c|c|c|}
\hline $\begin{array}{c}\text { Steric } \\
\text { Const } \\
\quad C \\
\end{array}$ & $\begin{array}{l}j=1 \\
k=1\end{array}$ & $\begin{array}{l}j=2 \\
k=1\end{array}$ & $\begin{array}{l}\mathbf{j}=\mathbf{3} \\
\mathbf{k}=\mathbf{1}\end{array}$ & $\begin{array}{l}j=6 \\
k=1\end{array}$ & $\begin{array}{l}j=7 \\
k=1\end{array}$ & $\begin{array}{l}\mathbf{j}=\mathbf{1 0} \\
\mathbf{k}=1\end{array}$ & $\begin{array}{l}j=11 \\
k=1\end{array}$ & $\begin{array}{l}j=1 \\
k=6\end{array}$ & $\begin{array}{l}j=1 \\
k=7\end{array}$ & $\begin{array}{c}\mathrm{j}=1 \\
\mathrm{k}=12\end{array}$ & $\begin{array}{c}j=1 \\
k=13\end{array}$ & $\begin{array}{l}j=7 \\
k=6\end{array}$ \\
\hline \multirow[t]{2}{*}{$V^{\mathrm{a}}$} & 0.950 & $\underline{0.952}$ & 0.914 & 0.925 & 0.937 & 0.897 & 0.948 & 0.907 & 0.945 & 0.922 & 0.951 & 0.888 \\
\hline & $>0.12$ & 0.12 & $>0.12$ & $>0.12$ & 0.12 & 0.05 & $>0.09$ & $>0.12$ & $>0.12$ & $>0.09$ & 0.09 & $>0.12$ \\
\hline \multirow[t]{2}{*}{$1 / S^{\prime}$} & 0.839 & 0.831 & 0.808 & $\underline{0.891}$ & 0.814 & 0.700 & 0.828 & 0.801 & 0.863 & 0.830 & 0.815 & 0.775 \\
\hline & 0.12 & $>0.12$ & $>0.12$ & 0.12 & $>0.12$ & 0.06 & $>0.09$ & $>0.12$ & $>0.12$ & $>0.09$ & $>0.09$ & $>0.12$ \\
\hline \multirow[t]{2}{*}{$\log S^{*}$} & $\underline{0.994}$ & 0.992 & 0.990 & 0.984 & $\underline{0.994}$ & 0.949 & 0.992 & 0.976 & 0.978 & 0.987 & 0.989 & 0.884 \\
\hline & 0.07 & 0.06 & 0.08 & 0.04 & 0.09 & 0.05 & 0.05 & 0.09 & 0.06 & $>0.09$ & 0.08 & 0.08 \\
\hline
\end{tabular}

The steric constants $v[6], \Xi[7]$ and $V^{\mathrm{a}}[9]$ were derived from van der Waals radii and other non-empirical structural parameters. In general, early calculated steric parameters are not very accurate. Among them, Charton's $v$ constant is significantly consistent with our $\Omega_{\mathrm{j}, \mathrm{k}}\left(R_{\mathrm{c}}\right)$. Its $r$ vs. $R_{\mathrm{c}}$ plots showed that $v$ have nearly the same accuracy and extent of application as $E_{\mathrm{s}}{ }^{\mathrm{c}}$, nevertheless the very simple and bold approximation during the process of calculation. Beckhaus's $\phi_{\mathrm{f}}$ is known as the steric constant suitable to measure so-called F-strain. Our analysis showed that the crowdedness hindering the access from the direction of $\mathrm{C}_{\alpha}-\mathrm{X}$ axis is the most important factor to determine the $\phi_{\mathrm{f}}$ value. Apparently contrary to F-strain, this implies that the backward crowding controls the steric energy of the system. However, this apparent inconsistency can be rationalized if we consider the model on which $\phi_{\mathrm{f}}$ was evaluated. In the model $\mathrm{R}-\mathrm{CMe}_{3}\left(=\mathrm{R}^{1} \mathrm{R}^{2} \mathrm{R}^{3} \mathrm{C}_{\alpha}-\mathrm{CMe}_{3}\right)$ molecule which takes the staggered conformation preferably, three substituents $\mathrm{R}^{1}, \mathrm{R}^{2}$, and $\mathrm{R}^{3}$ are pushed backward by three methyl groups of the bulky tert-butyl moiety. Steric crowdedness can be relieved if the backward direction (measurable by $\Omega_{6,1}$ ) is not crowded. These three methyl groups come just into the intersecting space of the three $\alpha$-substituents $\mathrm{R}^{1}, \mathrm{R}^{2}$, and $\mathrm{R}^{3}$, implying the importance of the steric crowdedness in between the two $\alpha$-substituents. In accord with this deduction, $\phi_{\mathrm{f}}$ becomes very dependent on $\Omega_{1,13}$.

Geometrical parameter $S$ (or $S^{\prime}$ ) [10] which was defined on the basis of the solid angle (in steradian) of substituents is very similar to our $\Omega_{\mathrm{s}}$, since both intended to estimate the steric hindrance by the solid angle of the shadow of substituent R. Nevertheless, $S$ itself was not a very good steric substituent constant, even if $1 / S$ ' showed a good linear correlation with $E$ s in a certain extent. As pointed out in relation to our $\Omega_{\mathrm{s}}$, the logarithm of the solid angle of the space open to the access of reagent should be a best steric constant in LFER. From this point of view, $\log _{10}\left(4 \pi-S^{\prime}\right)$ should be as good a steric constant as our $\log _{10}\left(1-\Omega_{\mathrm{s}}\right)$. As given in the last row of Table 3 , it is very closely correlated with our isotropic $\Omega_{\mathrm{s}}$ $\left(\Omega_{1,1}\right)$.

In conclusion, the statistical analysis employing the set of $\Omega_{\mathrm{j}, \mathrm{k}}\left(R_{\mathrm{c}}\right)$ is very effective in analyzing the steric effect on the reaction. The calculation of $\Omega_{\mathrm{j}, \mathrm{k}}\left(R_{\mathrm{c}}\right)$ involves several variable parameters useful for the diagnosis of the character of steric effect. Implications of the parameters controlling the angular weight-functions $(\mathrm{j}, \mathrm{k})$ and the size of reaction center atom $\mathrm{X}\left(R_{\mathrm{c}}\right)$ are discussed in this paper. 


\section{References and Notes}

1. (a) Taft, Jr. R. W. J. Am. Chem. Soc. 1952, 74, 2729; (b) Taft, Jr. R. W. J. Am. Chem. Soc.1952, 74, 3120; (c) Taft, Jr. R. W. "Steric Effects in Organic Chemistry," Newman, M. S., Ed.; John Wiley \& Sons, Inc.: New York, 1956.

2. (a) Hammett, L. P. J. Am. Chem. Soc. 1937, 59, 96; (b) Jaffe, H. H. Chem. Revs. 1953, 53,191.

3. (a) MacPhee, J. A.; Panaye, A.; Dubois, J.-E. Tetrahedron Lett. 1978, 3293; (b) MacPhee, J. A.; Jamal, M.; Guillaume, P.; Dubois, J.-E. Tetrahedron Lett. 1979, 1115.

4. (a) Hancock, C. K.; Meyers, E. A.; Yager, B. J. J. Am. Chem. Soc. 1961, 83, 4211; (b) Rosado-Lojo, O.; Hancock, C. K.; Danti, A. J. Org. Chem. 1966, 31, 1899.

5. Eq. 4 of ref. $4 \mathrm{~b}$.

6. (a) Wipke, W. T.; Gund, P. J. Am. Chem. Soc. 1974, 96, 299; 1976, 98, 8197; (b) DeTar, D. F.; Tenpas, C. J. J. Am. Chem. Soc. 1976, 98, 4567 ,7903; (c) White, D.; Taverner, B. C.; Leach, P. G. L.; Coville, N. J. J. Comput. Chem. 1993, 14, 1042.

7. (a) Charton, M. J. Am. Chem. Soc. 1969, 91, 615; (b) Charton, M. J. Am. Chem. Soc. 1975, 97, 1552 .

8. Kier, L. B. Quant. Struct. -Act. Relat., 1987, 6, 177.

9. Beckhaus, H. -D. Angew. Chem. Int. Ed. Engl. 1978, 17, 593.

10. Meyer, A. Y. J. Chem. Soc. Perkin Trans. II 1986, 1567.

11. Chauvin, R.; Kagan H. B. Chirality 1991, 242.

12. (a) Komatsuzaki, T.; Sakakibara, K.; Hirota, M. Tetrahedron Lett. 1989, 30, 3309; (b) Hirota, M.; Sakakibara, K.; Komatsuzaki, T.; Akai, I. Computer \& Chem. 1991, 15, 241; (c) Komatsuzaki, T.; Akai, I.; Sakakibara, K.; Hirota, M. Tetrahedron 1992, 48, 1539; (d) Ishizawa, J.; Sakakibara, K.; Hirota, M. Bull. Chem. Soc. Jpn. 1996, 69, 1003.

13. (a) Hirota, M.; Sakakibara, K.; Yuzuri, T.; Kuroda, S. J. Phys. Org. Chem., 2001, 14, 788; (b)Akai, I.; Kuroda, S.; Sakakibara, K.; Hirota, M. J. Phys. Org. Chem. 1995, 8, 791.

14. Area of the shadow of substituent $(\sigma)$ is dependent on many other parameters such as $r_{\mathrm{r}}, r_{\mathrm{s}}, T$ etc. The radius of reagent atom $\left(r_{\mathrm{r}}\right)$ has been shown to be rather insensitive to the $\Omega$ s value; thus we assigned $r_{\mathrm{r}}=0.05 \mathrm{~nm}$ throughout this work. Small perturbation of $\Omega$ s value can be originated from the choice of other parameters, but it never causes a serious inaccuracy of $\Omega$ s. See refs. 12 and13.

15. Angles $\theta$ and $\phi$ are defined on the basis of the polar coordinate system; its origin occupies the center of $\mathrm{X}$ atom and its axis lies on the $\mathrm{C}_{\alpha}-\mathrm{X}$ bond, and its positive end directs to the substituent from $\mathrm{X}$.

16. (a) Allinger, N. L.; Yuh, Y. H.; Lii, J.-H. J. Am. Chem. Soc. 1989, 111, 8551; (b) Allinger, N. L.; Lii, J.-H. J. Am. Chem. Soc. 1989, 111, 8566; (c) Allinger, N. L.; Lii, J.-H. J. Am. Chem. Soc. 1989, 111, 8576.

17. (a) Smith,H. A. J. Am. Chem. Soc. 1939, 61, 254; 1940, 62, 1136; (b) Smith, H. A.; Steele, J. H. J. Am. Chem. Soc. 1941, 63, 3466; (c) Evans, D. P.; Gordon, J. J.; Watson, H. B. J. Chem. Soc. 1938, 1439; (d) Bolton, P. D. Aust. J. Chem. 1966, 19, 1013; 1969, 22, 527; 1971, 24, 471; (e) Bolton, P. D.; Jackson, G. L. Aust. J. Chem., 1971, 24, 969; (f) Tommila, A. Ann. Acad. Sci. Fenn. Ser. A, 1942, 59, 3; (g) Norris, J. F.; Ashdown, A. A. J. Am. Chem. Soc. 1925, 47, 837; (h) Norris, J. F.; Cortese, F. J. Am. Chem. Soc. 1927, 49, 2640; (i) Bolton, P. D.; Ellis, J.; Frier, R. D. Nancarrow P. C.; Aust. J. 
Chem. 1972, 25, 303. (j) Fukuto, T. R.; Metcalf R. L.; J. Am. Chem. Soc. 1959, 81, 372; (k) Shimizu, N.; Takesue, N.; Yamamoto, A.; Tsutsumi, T.; Yasuhara, S.; Tsuno, Y. Chem. Lett. 1992,1263; (1) Shimizu, N.; Takesue, N.; Yasuhara, S.; Inazu, T. Chem. Lett. 1993, 1807; (m) Ozawa, S.; Ishii, S.; Hirata, K.; Hirose, M.; Kagaku-no-Ryoiki-Zokan, 1982, No. 136, 153 (Nanko-do Publ. Co.).

(C) 2005 by MDPI (http://www.mdpi.org). Reproduction is permitted for noncommercial purposes. 